\title{
Editorial to Special Issue on Evolutionary Computation in Dynamic and Uncertain Environments
}

Address(es) of author(s) should be given

Many real-world optimization problems, e.g., hardware design and job shop scheduling, are subjected to dynamic and uncertain environments that are often impossible to avoid in practice. For instance, the fitness function is uncertain or noisy as a result of simulation/measurement errors or approximation errors. In addition, the design variables or environmental conditions may also perturb or change over time. For these dynamic and uncertain optimization problems, the objective of the evolutionary algorithm (EA) is no longer to simply locate the global optimum solution, but to continuously track the optimum in dynamic environments, or to find a robust solution that operates optimally in the presence of uncertainties. This poses serious challenges to conventional EAs. However, conventional EAs with proper enhancements are still good tools of choice for optimization problems in dynamic and uncertain environments. This is because EAs are basically inspired by principles of natural evolution, which takes place in the ever-changing dynamic and uncertain environments in nature.

Handling dynamic and uncertain optimization problems in evolutionary computation has received increasing research interests over recent years due to its importance in practice. A variety of methods have been reported across a broad range of application backgrounds. This special issue aims to reflect the most recent advances, present sophisticated real-world applications, and explore future research directions in the field. Totally, we have received 14 submissions to this special issue, which cover a broad range of topics relevant to evolutionary computation in dynamic and uncertain environments. After a peer-review process, four papers of high quality are finally selected for publication in this special issue. These four papers represent the theoretical analysis, algorithm design, and application research in evolutionary computation under dynamic and uncertain environments.

In the first paper, Hans-Georg Beyer and Silja Meyer-Nieberg investigate the self-adaptation behaviour of $(1, \lambda)$-evolution strategies (ES) on the noisy sphere model. The steady state behaviour of ES is analyzed theoretically for constant and 
scaled noise strength scenarios and compared to the real ES runs. An explanation is given for the random walk like behaviour of the mutation strength in the vicinity of the steady state. It is shown to be a peculiarity of the $(1, \lambda)$-ES, which does not affect intermediate recombination strategies.

The second paper by Stefan Janson and Martin Middendorf addresses both noisy and dynamic environments using a partitioned hierarchical particle swarm optimization (PH-PSO). PH-PSO maintains a hierarchy of particles that are partitioned into sub-swarms when the environment changes and are rejoined after a proper time. To reduce the number of re-evaluations a new method is proposed which uses the hierarchy to find a subset of particles for which re-evaluations are particularly important. This paper also proposes detecting problem changes in the presence of noise based on the observation of changes in the swarm hierarchy. PHPSO has empirically proven efficient in addressing noisy and dynamic environments.

Yao Wang and Mark Wineberg contribute the third interesting paper, focusing on genetic algorithms (GAs) in dynamic environments. In their work, two evolvability measures based on the improvement in fitness and the amount of genotypic changes respectively, are proposed. These evolvability measures are integrated into the selection process of the GA via sub-populations to promote the evolvability of the GA in dynamic environments. Experimental study shows the efficiency of their evolvability promoted GA in dynamic environments.

The final paper by Dudy Lim et al. describes an inverse multi-objective robust evolutionary (IMORE) design methodology for robust design optimization. In contrast to conventional robust optimization, their approach avoids making assumptions about the uncertainty structure in formulating the optimization process, to avoid convergence to erroneous designs with catastrophic consequences. Adaptation strategies are also introduced in the paper to enhance the efficiency of IMORE. Empirical results show that the proposed methodology is able to efficiently achieve a set of design solutions with non-dominated nominal and robustness performance.

Generally speaking, this special issue fulfils the original aims quite well. The four papers collected in this special issue represent a great variety of work in the area of evolutionary computation under dynamic and uncertain environments. We hope that the publication of this special issue will further promote this emerging research field. Finally, we would like to thank the authors and reviewers for their contributions to this special issue. And particularly we would like to thank Professor Wolfgang Banzhaf for his great help to make this special issue a great success.

\author{
Shengxiang Yang \\ Yew-Soon Ong \\ Yaochu Jin
}

